University of Montana

ScholarWorks at University of Montana

$11-2010$

\title{
Hidden Consequences of Living in a Wormy World: Nematode- Induced Immune Suppression Facilitates Tuberculosis Invasion in African Buffalo
}

\author{
Vanessa O. Ezenwa \\ University of Montana - Missoula
}

Rampal S. Etienne

Gordon Luikart

University of Montana-Missoula, gordon.luikart@mso.umt.edu

Follow this and additional works at: https://scholarworks.umt.edu/biosci_pubs

Part of the Biology Commons

Let us know how access to this document benefits you.

\section{Recommended Citation}

Ezenwa, Vanessa O.; Etienne, Rampal S.; and Luikart, Gordon, "Hidden Consequences of Living in a Wormy World: Nematode-Induced Immune Suppression Facilitates Tuberculosis Invasion in African Buffalo" (2010). Biological Sciences Faculty Publications. 46.

https://scholarworks.umt.edu/biosci_pubs/46

This Article is brought to you for free and open access by the Biological Sciences at ScholarWorks at University of Montana. It has been accepted for inclusion in Biological Sciences Faculty Publications by an authorized administrator of ScholarWorks at University of Montana. For more information, please contact scholarworks@mso.umt.edu. 


\title{
Hidden Consequences of Living in a Wormy World: Nematode-Induced Immune Suppression Facilitates Tuberculosis Invasion in African Buffalo
}

\author{
Vanessa O. Ezenwa, ${ }^{1, *}$ Rampal S. Etienne, ${ }^{2}$ Gordon Luikart, ${ }^{1,3}$ Albano Beja-Pereira, ${ }^{3}$ and \\ Anna E. Jolles ${ }^{4}$
}

1. Division of Biological Sciences, University of Montana, Missoula, Montana 59812; 2. Community and Conservation Ecology Group, University of Groningen, Haren, The Netherlands; 3. Centro de Investigaçao em Biodiversidade e Recursos Geneticos (CIBIO-UP), Universidade do Porto, 4485-661 Vairão, Portugal; 4. Department of Biomedical Sciences, College of Veterinary Medicine, Oregon State University, Corvallis, Oregon 97331

Submitted December 24, 2009; Accepted July 21, 2010; Electronically published September 17, 2010

Online enhancements: appendix, table, figures.

ABStract: Most hosts are infected with multiple parasites, and responses of the immune system to co-occurring parasites may influence disease spread. Helminth infection can bias the host immune response toward a T-helper type 2 (Th2) over a type 1 (Th1) response, impairing the host's ability to control concurrent intracellular microparasite infections and potentially modifying disease dynamics. In humans, immune-mediated interactions between helminths and microparasites can alter host susceptibility to diseases such as HIV, tuberculosis (TB), and malaria. However, the extent to which similar processes operate in natural animal populations and influence disease spread remains unknown. We used cross-sectional, experimental, and genetic studies to show that gastrointestinal nematode infection alters immunity to intracellular microparasites in free-ranging African buffalo (Syncerus caffer). Buffalo that were more resistant to nematode infection had weaker Th1 responses, there was significant genotypic variation in nematode resistance, and anthelminthic treatment enhanced Th1 immunity. Using a disease dynamic model parameterized with empirical data, we found that nematode-induced immune suppression can facilitate the invasion of bovine TB in buffalo. In the absence of nematodes, TB failed to invade the system, illustrating the critical role nematodes may play in disease establishment. Our results suggest that helminths, by influencing the likelihood of microparasite invasion, may influence patterns of disease emergence in the wild.

Keywords: disease emergence, genetic resistance, immunity, nematode, tuberculosis, Syncerus caffer.

\footnotetext{
* Corresponding author. Present address: Odum School of Ecology and Department of Infectious Diseases, College of Veterinary Medicine, University of Georgia, Athens, Georgia 30602; e-mail: vezenwa@uga.edu.

Am. Nat. 2010. Vol. 176, pp. 613-624. (c) 2010 by The University of Chicago. 0003-0147/2010/17605-51827\$15.00. All rights reserved.

DOI: $10.1086 / 656496$
}

Introduction

Parasite-parasite interactions may be a powerful force shaping patterns of infectious disease spread in both humans and animals (Hotez et al. 2006; Telfer et al. 2008). Recently, considerable attention has focused on the role parasite coinfection might play in disease dynamics and emergence (Abu-Raddad et al. 2006; Lloyd-Smith et al. 2008). In humans, helminth infections have been linked to changes in the susceptibility to and progression of a variety of microparasitic diseases, including HIV/AIDS, tuberculosis, and malaria (e.g., Bentwich et al. 1999; Druilhe et al. 2005; Elias et al. 2006). Because helminthinduced changes in microparasite susceptibility and progression may significantly alter the rate at which hosts acquire and/or transmit microparasites, helminth coinfection could be an important factor influencing microparasite dynamics (Graham et al. 2007; Fenton 2008). In particular, because helminths are among the most common parasites of free-living animals (Poulin 2007), they may play an extensive yet underappreciated role in shaping disease emergence patterns in natural populations.

Helminth-microparasite interactions are thought to be driven in large part by mutually inhibitory effects of the T-helper type 1 (Th1) and type 2 (Th2) subsets of the vertebrate immune system. Thl cells produce messenger molecules (cytokines) that play a key role in amplifying immune effector mechanisms directed against intracellular microparasites (e.g., viruses, some bacteria and protozoa), while Th2 cells produce cytokines that promote mechanisms directed against macroparasites (e.g., helminths; Mosmann and Sad 1996). Because both classes of cytokines suppress production of the reciprocal type, helminthinduced Th2 responses can impair immunity to intracel- 
lular microparasites by inhibiting simultaneous Thl responses (Else and Finkelman 1998). For example, studies of the rodent immune response to a variety of helminth parasites have shown that antihelminth immunity is generally characterized by upregulation of Th2 responses, including interleukin-4 (IL-4) production and eosinophils, and downregulation of Th1 responses, including interferon $\gamma(\mathrm{IFN} \gamma)$ production (Finkelman et al. 1997; O'Neill et al. 2000; Bleay et al. 2007). Thus, the end result of chronic helminth infection can be the suppression of host protective immunity against coinfecting microparasites (e.g., Brady et al. 1999; La Flamme et al. 2002; Su et al. 2005). Recently, the role of helminths in stimulating regulatory $\mathrm{T}$ cells ( $\mathrm{T}$ regs) that dampen the activity of both Thl and Th2 cells via the action of immunoregulatory cytokines has also been described, suggesting an additional pathway by which immune-mediated helminth-microparasite interactions might occur during coinfection (Maizels et al. 2004). In either case, these interactions mean that a preexisting macroparasite infection may strongly influence the trajectory of secondary microparasite infections in coinfected hosts, in terms of disease susceptibility, intensity, and/or duration of infection. While experimental studies support the idea that concurrent helminth infection can influence the host immune response to microparasites ( $\mathrm{Su}$ et al. 2005; Ayash-Rashkovsky et al. 2007), most of this work has been restricted to lab animals, providing very little context for understanding how important such within-host processes are in natural populations and how they might scale up to influence broadscale ecological patterns of infectious disease in the wild.

In this study, we investigated the links between immunemediated parasite interactions and disease invasion in freeranging African buffalo (Syncerus caffer) in HluhluweiMfolozi Park, South Africa. These buffalo are infected by both gastrointestinal nematodes and bovine tuberculosis (Mycobacterium bovis; TB), but TB is exotic to the system and was first described in Hluhluwe-iMfolozi Park in 1986 (Michel et al. 2006). Our previous work in this system has shown that nematode and TB infections are negatively correlated with one another at the individual, among-herd, and population levels, a phenomenon that may be driven, at least in part, by cross-regulated immune responses (Jolles et al. 2008). However, documentation of a direct connection between nematode infection and a trade-off in Th1 immunity is lacking in buffalo or any other wild vertebrate (Bradley and Jackson 2008). Furthermore, it is unclear whether such immunological effects are sufficient to modify disease dynamics in nature. Here, we used a combination of cross-sectional and experimental studies to demonstrate that nematode infection impairs the host Th1 response under natural conditions. We also tested for genetic correlates of nematode resistance to examine whether worm-induced Thl suppression is enhanced among constitutively more resistant hosts. Finally, we used a disease dynamic model parameterized with empirical and genetic data to show that in the presence of an immunological trade-off, nematodes are capable of altering TB invasion success. Specifically, we tested the following predictions: (1) resistance to nematode infection is associated with reduced Th1 immunity in both TB-negative and TBpositive hosts; (2) this effect is inducible, and as such, experimental removal of nematodes enhances Th1 immune function; (3) constitutive genetic differences in nematode resistance contribute to the immune trade-off; and (4) given nematode infection-induced Th1-Th2 trade-offs and underlying fixed genetic differences in nematode resistance, nematodes can facilitate TB invasion.

\section{Empirical Methods}

\section{Study System}

Five hundred and forty-one African buffalo were captured and sampled in conjunction with the Bovine Tuberculosis Control Program at Hluhluwe-iMfolozi Park, South Africa. Animals were captured over a 2-week period in October 2005 (dry season, $n=281$ ) and again in May 2006 (wet season, $n=260$ ). Captures were carried out by Hluhluwe-iMfolozi Park management using a helicopter and funnel system to drive buffalo herds into a capture corral. Once corralled, buffalo were anesthetized for TB testing, during which we collected fecal and blood samples for parasite, immunological, and genetic analyses as well as information on animal age, sex, and body condition. For buffalo under the age of 2.5 years, body size and degree of horn development were used to assess age (Sinclair 1977). Animals between 2.5 and 5.5 years were aged by tooth eruption patterns (Jolles et al. 2005). For adults ( $>5.5$ years), tooth wear of the first incisor was used as an indicator of age (Jolles et al. 2005). Body condition was assessed using a condition-scoring index based on visual inspection and manual palpation of four areas of the body where buffalo store fat: ribs, spine, hips, and base of the tail. Each body part was given a score between 1 and 5, with a higher score reflecting greater fatness. The sum of all four scores was used as a composite measure of overall body condition (Ezenwa et al. 2009).

\section{Infection and Immunity Patterns}

To investigate associations between nematodes and host immune function, we evaluated nematode and TB infection status and measured immunological profiles for all study animals. Buffalo were tested for TB using a tuberculin skin test, with sensitivity $80.9 \%$ and specificity $90.2 \%$ 
(J. P. Raath, unpublished data). TB positives were culled as part of the TB control program, and TB severity was assessed post mortem by quantifying macroscopic lesions in the lungs and lymph nodes of the head and respiratory tract. Seventy-five percent of study animals with a positive skin test showed signs of TB lesions in one or more tissues, and overall severity was classified on a scale of $0-4$, as described by Jolles et al. (2005). Gastrointestinal nematode infection was assessed from fecal samples collected during capture. Samples were collected directly from the rectum of anesthetized animals and stored at $4^{\circ} \mathrm{C}$ for $0-5$ days until processing. Strongyle (Nematoda: Strongylida) nematode output in host feces was quantified using a modification of the McMaster fecal egg counting technique (Ezenwa 2003). Fecal egg counts (FECs) reflect the number, size, and fecundity of the worm population within a host and provide a relative index of the degree of control exerted by the host on these parasites (Gasbarre et al. 2001). In our system, nematode FEC was negatively correlated with eosinophils, a primary Th2 effector mechanism controlling helminth infections, and this pattern was robust to controlling for host age, sex, and capture period (ANCOVA [TB negatives only] log FEC: $F_{1,430}=4.27$, coefficient $=-0.021, P=.039$; age: $F_{1,430}=6.56$, coefficient $=-0.007, P=.011$; sex: $F_{1,430}=3.11, P=.079$; capture period: $\left.F_{1,430}=71.3, P=<.0001\right)$. We have also previously shown that worm-free buffalo have significantly higher eosinophil counts than worm-infected buffalo (Jolles et al. 2008). In combination, these results suggest that individuals with low FECs mount stronger Th2 immune responses to nematodes, and as such, we used FEC as an inverse measure of the degree of host resistance to nematodes.

We assayed host Th1 immune function by measuring interferon $\gamma$ (IFN $\gamma$ ) levels in blood. IFN $\gamma$ is a hallmark messenger molecule of the Th1 response, acting as a primary mediator of macrophage activation for killing intracellular pathogens (Abbas et al. 1996; Murphy et al. 2007). In particular, IFN $\gamma$ plays a necessary role in the protective immune response to TB (Flynn et al. 1993; Welsh et al. 2005). Blood samples were collected from the jugular vein of individual buffalo into $10-\mathrm{mL}$ heparin tubes. All samples were taken during the first immobilization before animals were injected with tuberculin antigen for TB skin testing. Following a standard whole-blood gamma interferon assay protocol (Wood and Jones 2001; Schiller et al. 2009), we quantified baseline circulating levels of IFN $\gamma$ (hereafter referred to as baseline IFN $\gamma$ ) by incubating whole blood at $37^{\circ} \mathrm{C}$ for $28 \mathrm{~h}$ and then assaying IFN $\gamma$ concentrations in the supernatant plasma using the BOVIGAM enzymelinked immunosorbent assay (ELISA) kit (Prionics, Switzerland). For all assays, incubation occurred within $8 \mathrm{~h}$ of blood collection, and after incubation, plasma was im- mediately frozen and kept at $-20^{\circ} \mathrm{C}$ until processing. Optical densities were read at $450 \mathrm{~nm}\left(\mathrm{OD}_{450}\right)$. We also assessed host responsiveness to in vitro stimulation with Mycobacterium bovis antigen (bovine purified protein derivative $[\mathrm{PPDB}]$, supplied by the Animal Sciences Group, Wageningen, Netherlands) by adding $30 \mu \mathrm{L}$ of PPDB to blood before incubation, with further analysis as described above. The host response to PPDB challenge was quantified as the proportionate change in $\mathrm{OD}_{450}$ (IFN $\gamma$ PPDB $\mathrm{OD}_{450} / \mathrm{IFN} \gamma$ baseline $\mathrm{OD}_{450}$; hereafter referred to as the IFN $\gamma$ PPDB response). This assay, widely used for bovine TB testing, measures the host's cell-mediated response to antigenic stimulation and differentiates between TBpositive and TB-negative animals on the basis of a predominant antigen-specific response in TB-infected hosts (Wood and Jones 2001; Grobler et al. 2002; Schiller et al. 2009).

\section{Anthelminthic Treatment Experiment}

To test for a causal effect of nematodes on Th1 immune function, we treated 57 captured TB-negative animals for nematodes in October 2005. Each treated animal was given a single dose of a slow-release Fenbendazole bolus (Panacur, Hoechst-Roussel, Netherlands), delivered orally to the rumen. A single Panacur bolus effectively eliminates nematode egg shedding for $\sim 160$ days in wild African buffalo (V. O. Ezenwa and A. E. Jolles, unpublished data). Animals of similar age and sex were designated as controls. Controls received nothing. All animals were marked with implantable transponder chips (Trovan) to facilitate individual identification on recapture. Host traits and IFN $\gamma$ levels were measured as described above.

\section{Genetic Analyses}

To test for an underlying genetic basis to nematode resistance in our study population, we preserved whole blood collected from buffalo captured in May 2006 on FTA cards (Whatman, Clifton, NJ). Dried cards were stored at room temperature until DNA extraction. DNA was extracted from 84 cards using the GenSolve DNA Recovery Kit as directed by the manufacturer (Whatman). Samples were genotyped at the BL4 microsatellite locus located upstream of the IFN $\gamma$ gene, a gene that has been associated with nematode resistance in sheep (Coltman et al. 2001). Forward primers were fluorescent labeled, and PCR products were separated by capillary electrophoresis on an automated sequencer (3130XL, Applied Biosystems). Alleles were scored using GeneMapper. Twenty percent of samples were regenotyped to verify data quality, and no differences or errors were detected. We tested for deviations from 
Hardy-Weinberg proportions using exact tests implemented in Genepop 3.4 (Raymond and Rousset 1995).

\section{Statistical Analyses}

We compared baseline IFN $\gamma$ and IFN $\gamma$ PPDB responses in TB-negative and TB-positive buffalo using MannWhitney $U$-tests to assess the magnitude of the difference in Thl responses between the two groups. To normalize data distributions, the IFN $\gamma$ data were then transformed as appropriate for analyses within groups. For baseline IFN $\gamma$ in TB-negative and TB-positive animals, we used an inverse transformation and subtracted the transformed values from a constant to reinstate the original data set order. For IFN $\gamma$ PPDB responses in TB-positive animals, we $\log _{10}$ transformed the IFN $\gamma$ PPDB $\mathrm{OD}_{450}$ : IFN $\gamma$ baseline $\mathrm{OD}_{450}$ ratio. Efficacy of the transformations was evaluated by examining skewness, kurtosis, and Shapiro-Wilk's $W$ values (skewness and kurtosis $\leq|2| ; W \geq 0.95$ ) and by checking model residuals for normality.

To test whether nematode infection affected baseline Th1 immunity, we examined associations between nematode FEC and baseline IFN $\gamma$ in both TB-negative and TB-positive hosts. We used ANCOVAs with baseline IFN $\gamma$ as the dependent variable and FEC $\left(\log _{10}(\mathrm{FEC}+1)\right)$ as the main predictor. For the TB-negative model, we included host age, sex, body condition, and capture period (October vs. May) as covariates. For the TB-positive model, we added two additional covariates: body condition $\times$ FEC interaction (since we previously found body condition to decline in TB-nematode coinfected hosts; Jolles et al. 2008) and $\mathrm{TB}$ severity (since host IFN $\gamma$ levels can be strongly influenced by TB disease severity; Vordermeier et al. 2002). A model of similar structure was used to test the effect of nematode FEC on the IFN $\gamma$ PPDB response in TB-positive animals. We also examined associations between body condition and nematode FEC in TB negatives using an ANCOVA with body condition as the dependent variable and FEC, age, sex, and capture period as independent variables. For all these analyses, we excluded records from the second capture of all recaptured individuals to avoid pseudoreplication.

We tested whether parasite removal via anthelminthic treatment had effects on nematode FEC, baseline IFN $\gamma$, and body condition using a set of repeated-measures ANOVAs with treatment status as the between-subjects factor. We also examined whether parasite treatment effects on baseline IFN $\gamma$ were independent from effects on body condition by constructing an ANCOVA for postexperiment baseline IFN $\gamma$ that included preexperiment IFN $\gamma$, treatment status, and postexperiment body condition as covariates.

Finally, we used an ANCOVA to test associations be- tween BL4 alleles and nematode FEC. The model included the presence or absence of each allele as factors, along with host age, sex, and body condition. Capture period was not included as a covariate since genetic samples were collected only in May 2006. After linking genetic data to FEC levels in the May 2006 population, we used cutoffs derived from this analysis to classify all individuals caught across both capture periods into nematode-resistant phenotypes. We then tested for differences in baseline IFN $\gamma$ levels between TB-negative animals of different phenotypes (nematode resistant vs. nonresistant). To do this, we used an ANCOVA, and as with previous IFN $\gamma$ analyses, we controlled for host age, sex, body condition, and capture period.

\section{Empirical Results}

\section{Nematode Effects on Th1 Immunity in TB-Negative and TB-Positive Hosts}

There was no significant difference between TB-negative and TB-positive hosts in their baseline IFN $\gamma$ levels (MannWhitney $U=13,918, P=.216$; fig. $1 A$ ), but TB positives showed a greater response to PPDB stimulation compared with TB negatives $(U=3,790, P<.0001$; fig. $1 B)$. On the basis of these results, we tested for the effect of nematode FEC on baseline IFN $\gamma$ levels in both groups and on IFN $\gamma$ PPDB responses only in TB positives.

For TB-negative hosts, we found that individuals with lower FECs had significantly weaker baseline IFN $\gamma$ levels (table 1). IFN $\gamma$ levels were also significantly lower during the October capture period compared with the May capture period (table 1). For TB-positive hosts, age was the only factor that influenced baseline IFN $\gamma$ levels, with younger animals showing significantly lower levels (table $2)$. By contrast, TB severity had a significant effect on the IFN $\gamma$ PPDB response in TB positives, and the effects of FEC and body condition were marginally significant (table 2). PPDB responses were stronger in animals with more severe TB infections and those with higher nematode FECs and in better condition (table 2).

\section{Treatment Experiment}

We recaptured 17 study animals (four treated and 13 controls), and the interval between initial capture and recapture ranged from 202 to 229 days. At recapture, there was no significant difference in FEC between treated and control animals, although treated animals tended to have lower egg counts than controls (repeated-measures ANOVA; $F_{1,14}=0.424 ; P=.525 ;$ treated, $n=4: \log$ $\mathrm{FEC} \pm \mathrm{SE}=1.047 \pm 0.624$; controls, $n=12: 1.513 \pm$ 0.341 ). Baseline IFN $\gamma$ levels in the treated group increased significantly after treatment compared with untreated con- 


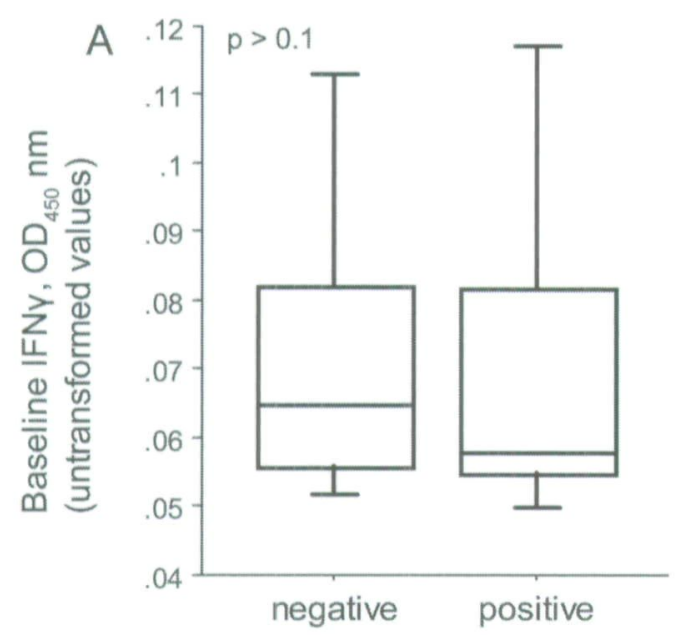

TB status

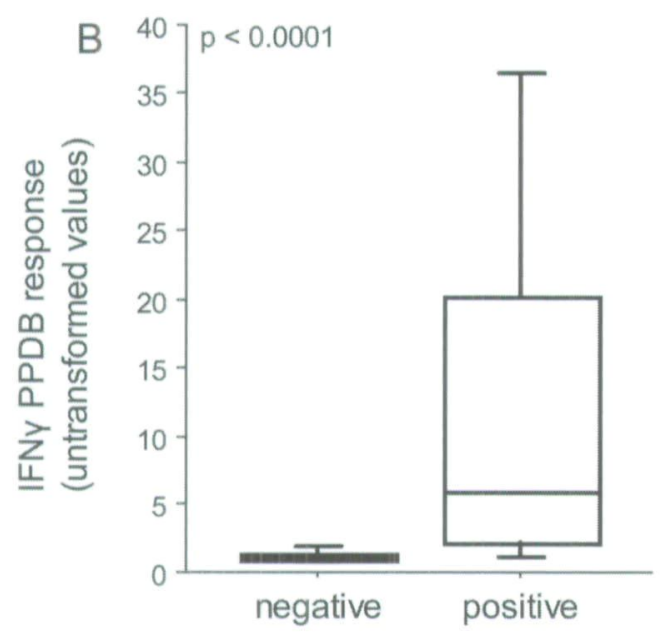

TB status

Figure 1: Differences in baseline interferon $\gamma(\operatorname{IFN} \gamma ; A)$ and IFN $\gamma$ bovine purified protein derivative (PPDB) response $(B)$ between tuberculosis (TB) skin test negative and positive buffalo. Boxes indicate the twentyfifth percentile, the median (fiftieth percentile), and the seventy-fifth percentile. Bars represent the tenth and ninetieth percentiles.

trols $\left(F_{1,15}=11.4, P=.0041\right.$; fig. $\left.2 A\right)$. There was also a significant overall increase in baseline IFN $\gamma$ from October 2005 to May $2006\left(F_{1,15}=26.9, P=.0001\right.$; fig. $\left.2 A\right)$. Because cross-sectional data showed that individuals with higher egg counts were in poorer condition (ANCOVA; log FEC: $F_{1,460}=4.848, P=.0282 ;$ age: $F_{1,460}=38.35$, $P<.0001$; sex: $F_{1,460}=5.665, P=.0177$; capture period: $\left.F_{1,460}=338.54, P<.0001\right)$, we also tested the effect of anthelminthic treatment on host body condition. Consistent with this observation, body condition increased significantly in response to treatment $\left(F_{1,15}=10.5, P=.0056\right.$; fig. 2B). Condition also increased from October to May $\left(F_{1,15}=89.4, P<.0001\right.$; fig. $\left.2 B\right)$. When we examined whether increases in body condition in response to treatment could account for the observed treatment-induced changes in IFN $\gamma$, we found that treatment status, but not posttreatment body condition, significantly explained posttreatment baseline IFN $\gamma$ (ANCOVA; treatment status: $F_{1,13}=7.18, P=.0189$; posttreatment body condition: $F_{1,13}=0.008 ; \quad P=.9291 ;$ pretreatment $\operatorname{IFN} \gamma: F_{1,13}=$ $1.48, P=.2455)$.

\section{Genetic Correlates of Nematode Resistance and Classification of Resistant and Nonresistant Phenotypes}

We identified seven alleles at the BL4 locus in a subset of animals from our study population, and these alleles ranged in frequency from $3.7 \%$ to $41.5 \%(n=84)$. The observed proportion of heterozygotes $\left(H_{\mathrm{o}}\right)$ was $0.683 \mathrm{com}$ pared with an expected value $\left(H_{\mathrm{e}}\right)$ of 0.754 , with no evidence of a departure from Hardy-Weinberg proportions ( $F_{\mathrm{IS}}$ [inbreeding coefficient of individuals within subpopulations] $=0.095, P=.201)$. Of the seven BL4 alleles, one (BL4-169) was significantly associated with nematode FEC $\left(F_{1,73}=8.776, P=.004\right)$. Host FEC was significantly higher in the presence of BL4-169 than in its absence (present $[1]: n=56, \log \mathrm{FEC} \pm \mathrm{SE}=1.956 \pm 0.241$; absent $[0]: n=28, \log \mathrm{FEC} \pm \mathrm{SE}=1.202 \pm 0.182$; fig. $3 A$ ). Given evidence of a link between host genotype and nematode FEC, we used the mean untransformed FEC value of individuals lacking allele $169(n=462$; mean value: 275 nematode eggs per gram feces [EPG]) as a threshold for classifying all TB-negative study animals into nematode-resistant (low FEC: $\leq 275 \mathrm{EPG}, n=194$ ) versus nonresistant (high FEC: $>275$ EPG, $n=268$ ) phenotypes. When we tested for differences in Th1 immunity between these two groups, we found that discrete differences in resistance level (low vs. high FEC) were associated with significant differences in baseline IFN $\gamma$ (ANCOVA [TB negatives only]; FEC level: $F_{1,456}=7.784, P=.005$; age: $F_{1,456}=1.041, P=.308 ;$ sex: $F_{1,456}=1.288, P=.257$;

Table 1: Predictors of baseline IFN $\gamma$ levels in TB-negative buffalo

\begin{tabular}{lccc}
\hline Variable & Coefficient & $F$ & $P$ \\
\hline Age & .052 & 1.126 & .2892 \\
Sex: female & -.247 & 1.418 & .2344 \\
Capture period: October & -1.157 & 18.14 & $<.0001^{*}$ \\
Body condition & -.105 & 1.462 & .2273 \\
Nematode FEC & .418 & 5.58 & $.0186^{*}$ \\
\hline
\end{tabular}

Note: $n=462$. IFN $\gamma$, interferon $\gamma$; TB, tuberculosis; FEC, fecal egg count.

$$
\text { * } P<.05 \text {. }
$$


Table 2: Predictors of baseline IFN $\gamma$ and IFN $\gamma$ PPDB response levels in TB-positive buffalo

\begin{tabular}{|c|c|c|c|c|c|c|}
\hline \multirow[b]{2}{*}{ Variable } & \multicolumn{3}{|c|}{ Baseline IFN $\gamma$} & \multicolumn{3}{|c|}{ IFN $\gamma$ PPDB response } \\
\hline & Coefficient & $F$ & $P$ & Coefficient & $F$ & $P$ \\
\hline Age & -.442 & 5.027 & $.029^{* *}$ & .068 & 2.338 & .132 \\
\hline Sex: female & .217 & .075 & .785 & -.016 & .008 & .930 \\
\hline Capture period: October & -1.025 & 1.073 & .305 & .061 & .075 & .786 \\
\hline Body condition & -.472 & 1.216 & .275 & .191 & 3.908 & $.053^{*}$ \\
\hline Nematode FEC & -1.662 & .371 & .545 & 1.193 & 3.731 & $.058^{*}$ \\
\hline Nematode FEC $\times$ body condition & .071 & .132 & .718 & -.073 & 2.758 & .102 \\
\hline TB severity & -.046 & .005 & .942 & .374 & 6.745 & $.012^{\star * *}$ \\
\hline
\end{tabular}

Note: $n=65$. IFN $\gamma$, interferon $\gamma$; PPDB, bovine purified protein derivative; TB, tuberculosis; FEC, fecal egg count.

$* P<.1$.

** $P<.05$

body condition: $F_{1,456}=1.530, P=.217$; capture period: $\left.F_{1,456}=17.636, P<.0001\right)$. Low-FEC (nematode-resistant) individuals had lower IFN $\gamma$ levels than high-FEC (nonresistant) individuals (fig. $3 B$ ).

\section{Disease Dynamic Model: Methods}

We developed a simple deterministic model to explore the consequences of nematode-induced immune suppression for TB invasion in buffalo (fig. 4). We formulated the model as follows on the basis of our empirical results: buffalo were subdivided into two classes, nematode resistant (resistance is denoted with an asterisk) and nonresistant, to capture genotypic variation in fecal egg counts that also reflects differences in Th1 immunity (see fig. $3 B$; resistant $=$ low FEC and nonresistant $=$ high FEC). Each of these classes was further subdivided into nematode infected and susceptible (i.e., noninfected). Nematode exposure, analogous to the rate at which susceptible individuals become infected with nematodes, or the force of infection, was set as equal for both resistance classes $\left(\beta_{\mathrm{w}}=\beta_{\mathrm{w}}^{*}\right)$ and did not change in response to changes in the number of infected individuals. Once exposed to worms, all individuals mount an antinematode response, which results in depressed Th1 immune function and increased TB susceptibility. The antinematode response mounted by resistant individuals is stronger than the response mounted by nonresistant individuals. Thus, when buffalo are exposed to nematodes, TB transmission increases and nematode-resistant individuals have higher TB transmission rates than nonresistant individuals $\left(\beta_{\mathrm{tb}}=\right.$ $\left.\beta_{\mathrm{tb}}^{*}<\beta_{\mathrm{tb}}^{\mathrm{w}}<\beta_{\mathrm{tb}}^{\mathrm{w} *}\right)$. In the absence of nematodes, TB transmission in resistant and nonresistant genotypes is equivalent and lower than TB transmission in the presence of worms. We did not include an effect of nematodes on the rate at which TB-infected hosts transmit infection (i.e., TB infectiousness), given the weak association we found between nematode FEC and Th1 immunity in TB-positive animals (see table 1).
For simplicity, we did not track individual nematode burdens, even though macroparasite infections are typically modeled in this manner. Nematode exposure was assumed to be independent of buffalo herd size, since buffalo tend to be infected with many generalist nematode species and transmission is likely a function of contact with both conspecifics and heterospecifics (Ezenwa 2003). Nematode recovery occurred at a faster rate in the resistant class compared with the nonresistant class because resistant individuals are genetically predisposed to mount stronger antinematode responses $\left(\gamma_{\mathrm{w}}=\gamma_{\mathrm{w}, \mathrm{tb}}<\gamma_{\mathrm{w}, \mathrm{tb}}^{*}=\right.$ $\left.\gamma_{\mathrm{w}}^{*}\right)$. Because immunity to nematode infection is not complete (Gasbarre et al. 2001), hosts recovering from nematode infections were susceptible to reinfection. For TB, we assumed that transmission occurs via mass action. There is no evidence that animals recover from TB (Bengis 1999), so we did not include a term for TB recovery. Because we have no information on the effect of nematodes on buffalo mortality, we set the mortality rate for nematode-infected buffalo equal to the background mortality rate for disease-free individuals. The TB-induced mortality rate was assumed to be equal for both nematoderesistant and nonresistant individuals. The mortality rate for coinfected hosts was assumed to be higher on the basis of data showing that coinfection negatively affects host condition and may accelerate mortality (Jolles et al. 2008), and nematode-nonresistant individuals infected with both nematodes and TB had a higher mortality rate than nematode-resistant individuals $\left(\mu=\mu_{\mathrm{w}}<\mu_{\mathrm{tb}}=\mu_{\mathrm{tb}}^{*}<\mu_{\mathrm{w}, \mathrm{tb}}^{*}<\right.$ $\left.\mu_{\mathrm{w}, \mathrm{tb}}\right)$. Finally, in the absence of $\mathrm{TB}$, the total birth rate was set equal to the death rate so that population size $N$ was constant. Model parameters were largely derived from empirical data (table A1 in the online edition of the American Naturalist), and when we used arbitrarily selected values, we performed sensitivity analyses to assess the effect of our parameter choices on model output (see appendix in the online edition of the American Naturalist).

We used our model to test the effect of variation in the nematode transmission rate on the basic reproductive 
A

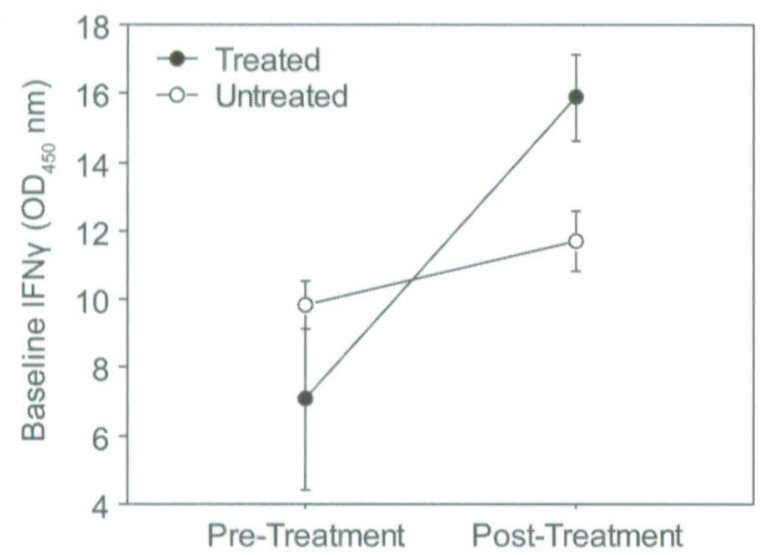

B

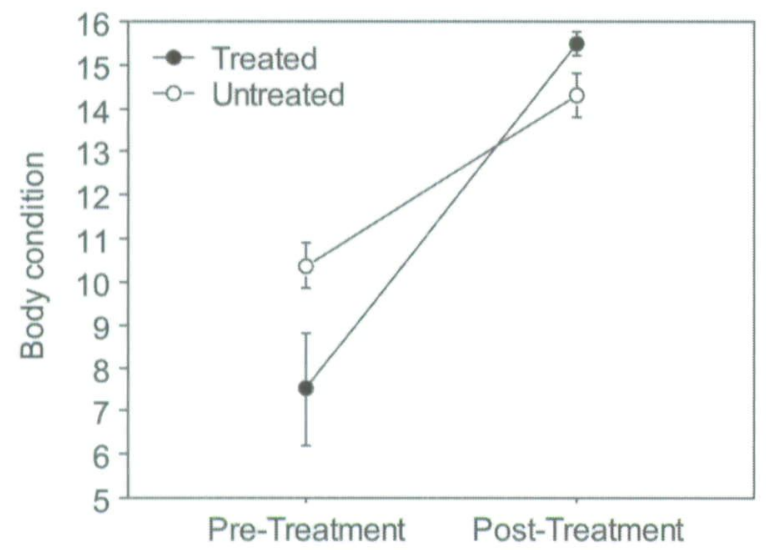

Figure 2: Effects of anthelminthic treatment on baseline interferon $\gamma$ $(\mathrm{IFN} \gamma)$ levels and body condition. $A, \mathrm{IFN} \gamma$ increased in response to anthelminthic treatment from October 2005 (pretreatment period, dry season) to May 2006 (posttreatment period, wet season). B, Body condition showed a similar response to treatment.

number $\left(R_{0}\right)$ of TB (for details on $R_{0}$ calculations, see appendix). $R_{0}$ is defined as the number of secondary infections arising from a single infection in a wholly susceptible population. A pathogen can invade a host population only if it is successfully transmitted from the index host to more than one new host; thus, invasion occurs only when $R_{0}>1$. We calculated the $R_{0}$ of TB for a range of nematode transmission rates $\left(\beta_{\mathrm{w}}\right)$ under two scenarios: $\mathrm{S}_{1}$, with the birth rate of nematode-resistant individuals $\left(b^{*}\right)$ set equal to the birth rate of nonresistant individuals (b); and $\mathrm{S}_{2}$, with $b^{*}=2 b$, reflecting the observed ratio of nematode-resistant to nonresistant individuals at the BL4 locus in our study population.

\section{Disease Dynamic Model: Results}

We found that TB failed to invade the buffalo population in the absence of nematodes (when $\beta_{w}=0, R_{0}<1$; fig.
5). However, with increasing nematode transmission, the $R_{0}$ of TB increased above 1, allowing for invasion. This effect of nematodes on TB $R_{0}$ was apparent in all model runs with $\beta_{\mathrm{tb}}^{\mathrm{w}} \geq 1.5 \times \beta_{\mathrm{tb}}$ (fig. A2 in the online edition of the American Naturalist), suggesting that a minimum nematode-induced TB transmission differential of 50\% (e.g., a change in the TB transmission rate from 0.1 to 0.15 among nematode-infected buffalo) is sufficient to facilitate TB invasion in our system. Both genotypic distributions we examined $\left(b^{*}=b\right.$ and $\left.b^{*}=2 b\right)$ showed qualitatively similar relationships between $\beta_{\mathrm{w}}$ and $R_{0}$. For example, at $\beta_{w}=0.05, \quad R_{0}\left(b^{*}=b\right)=1.009$ and $R_{0}\left(b^{*}=2 b\right)=$ 1.028 , but TB achieved a slightly higher maximum $R_{0}$ with a higher frequency of nematode-resistant individuals in the population (fig. 5).

A

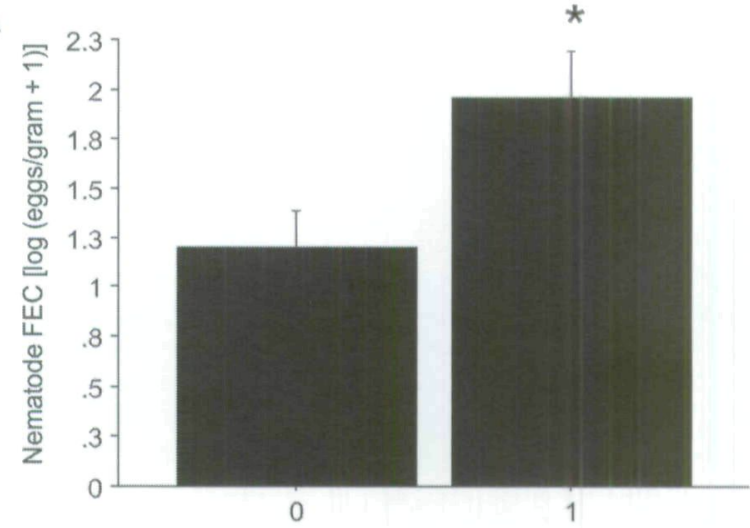

BL4-169

B

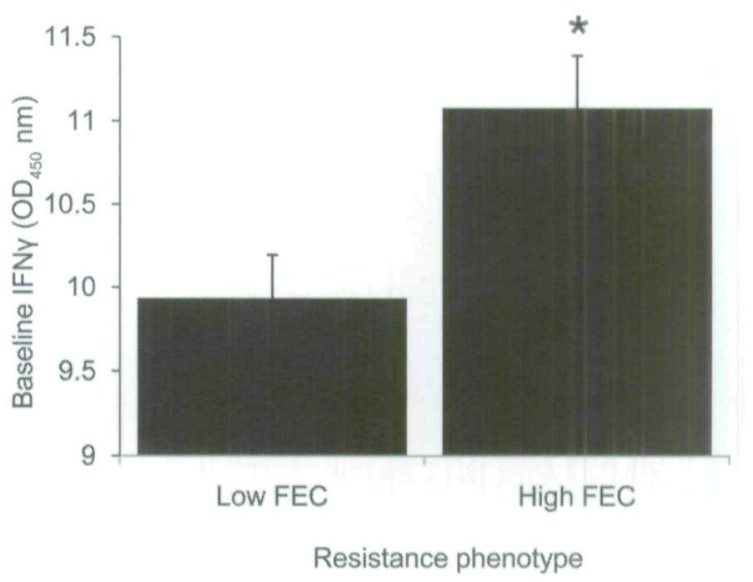

Figure 3: A, Association between the presence (1) or absence ( 0 ) of allele BL4-169 and nematode fecal egg count (FEC; mean \pm SE). B, Association between resistance phenotype and baseline interferon $\gamma$ (IFN $\gamma$; least squares mean $\pm \mathrm{SE}$ ). Resistance phenotype (low vs. high FEC) was classified on the basis of cutoff values determined from the relationship between BL4-169 presence/absence and nematode FEC. 


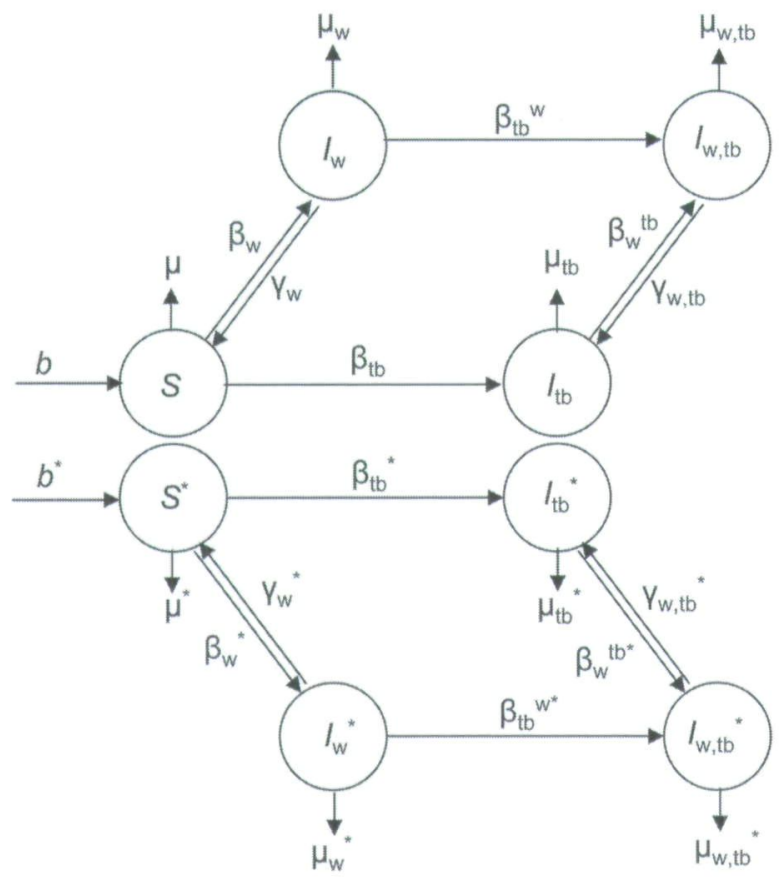

Figure 4: Schematic representation of the disease dynamic model. Hosts are partitioned into two classes on the basis of response to nematode infection: resistant and nonresistant (resistance is denoted with an asterisk). Both classes form a single homogenous population. Within each class, individuals fall into one of four groups: susceptible $\left(S / S^{*}\right)$, infected with worms only $\left(I_{\mathrm{w}} / I_{\mathrm{w}}^{*}\right)$, infected with tuberculosis (TB) only $\left(I_{\mathrm{tb}} / I_{\mathrm{tb}}^{*}\right)$, and coinfected $\left(I_{\mathrm{w}, \mathrm{tb}} / I_{\mathrm{w}, \mathrm{tb}}^{*}\right)$. Hosts are born at rate $b$ and move between groups via worm transmission $\left(\beta_{\mathrm{w}}\right)$, TB transmission $\left(\beta_{\mathrm{tb}}\right)$, and nematode recovery $\left(\gamma_{\mathrm{w}}\right)$. Hosts die at rate $\mu$. The relative value of transition rates depends on the host's infection status and worm resistance status. For the TB transmission rate, $\beta_{\mathrm{tb}}=\beta_{\mathrm{tb}}^{*}<\beta_{\mathrm{tb}}^{\mathrm{w}}<\beta_{\mathrm{tb}}^{\mathrm{w}^{*}}$. For the worm recovery rate, $\gamma_{\mathrm{w}}=\gamma_{\mathrm{w}, \mathrm{tb}}<\gamma_{\mathrm{w}, \mathrm{tb}}^{*}=\gamma_{\mathrm{w}}^{*}$. For the mortality rate, $\mu=\mu_{\mathrm{w}}<\mu_{\mathrm{tb}}=$ $\mu_{\mathrm{tb}}^{\mathrm{x}}<\mu_{\mathrm{w}, \mathrm{tb}}^{*}<\mu_{\mathrm{w}, \mathrm{t}}$. Parameter values: $\beta_{\mathrm{tb}}=0.1, \beta_{\mathrm{tb}}^{\mathrm{w}}=3 \times \beta_{\mathrm{tb}}, \beta_{\mathrm{tb}}^{\mathrm{w}^{*}}=$ $1.2 \times \beta_{\mathrm{tb}}^{\mathrm{w}}, \mu=\mu_{\mathrm{w}}=0.05, \mu_{\mathrm{tb}}=\mu_{\mathrm{tb}}^{*}=0.15, \mu_{\mathrm{w}, \mathrm{tb}}^{*}=1.03 \times \mu_{\mathrm{tb}}, \mu_{\mathrm{w}, \mathrm{tb}}=$ $1.09 \times \mu_{\mathrm{w}, \mathrm{tb}}^{*}, \gamma_{\mathrm{w}}=\gamma_{\mathrm{w}, \mathrm{tb}}=0.1, \gamma_{\mathrm{w}, \mathrm{tb}}^{*}=\gamma_{\mathrm{w}}^{*}=1.1 \times \gamma_{\mathrm{w}}$. Invasion criteria $\left(R_{0}\right)$ for TB were explored under a range of nematode transmission rates with $\beta_{\mathrm{w}}=\beta_{\mathrm{w}}^{*}$.

\section{Discussion}

Our study suggests that nematodes affect Th1 immune function in buffalo and that this effect may be sufficient to alter TB invasion success. Two lines of evidence point to a link between nematodes and Th1 immunity in our system. First, buffalo with higher egg counts had higher baseline IFN $\gamma$ levels; second, anthelminthic treatment enhanced IFN $\gamma$ production. We used fecal egg counts as an index of nematode resistance, an assumption upheld by data from our study population showing that individuals shedding nematode eggs had lower eosinophil counts (Jolles et al. 2008) and that higher egg counts were associated with lower eosinophil counts (see "Empirical Methods"). There is also ample evidence from domestic animals linking variation in FEC to the degree of host resistance (Gasbarre et al. 2001; Stear et al. 2007). Thus, the positive association we saw between FEC and baseline IFN $\gamma$ in TB-negative buffalo suggests that hosts with higher egg counts, who were poorer at controlling their nematode infections, produced more IFN $\gamma$. By contrast, hosts that exerted stronger control over nematodes had weaker Thl responses. This is consistent with observations from mouse models that hosts with poor nematode control (i.e., nonresistant hosts) show dominant Th1 responses in the presence of infection, whereas resistant individuals develop dominant Th2 profiles (Grencis 2001).

If Th1 suppression occurs in response to upregulation of antinematode immunity, then a reduction in nematode infection pressure should moderate these effects. Crucially, we found that anthelminthic treatment increased baseline levels of IFN $\gamma$, providing strong support for the idea that nematode exposure alters Thl immunity. Similarly, a recent helminth-malaria coinfection study in mice found that concurrent infection with the nematode Heligmosomoides polygyrus suppressed production of IFN $\gamma$ and impaired protective immunity against malaria, but anthelminthic treatment restored antimalarial immunity (Su et al. 2005). While our study did not examine the specific pathways by which nematode infection induces Thl suppression, recently it has become clear that Th1-Th2 crossregulation is not the sole pathway by which these effects can occur. For example, in the H. polygyrus malaria study (Su et al. 2005), immunosuppressive effects of nematode infection were induced via upregulation of the immunoregulatory cytokine transforming growth factor $\beta 1$. Regardless of the exact biochemical nature of the interaction,

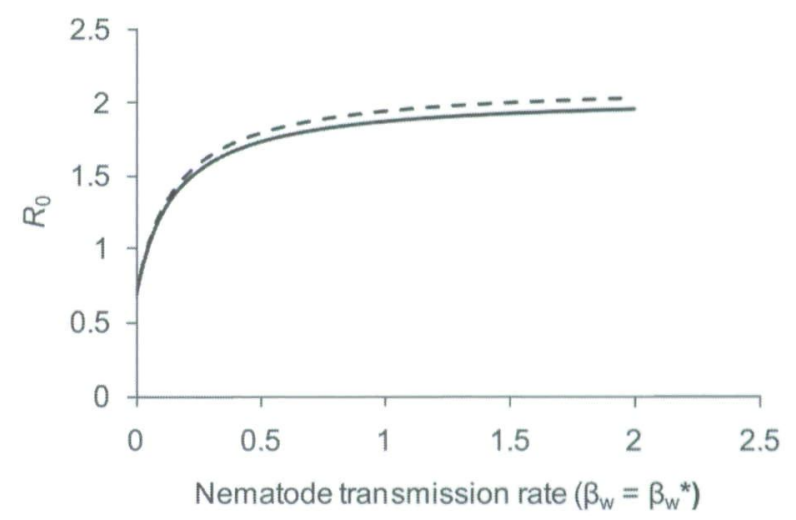

Figure 5: Model results showing changes in the basic reproductive number $\left(R_{0}\right)$ of tuberculosis at different levels of nematode transmission under two different scenarios. The solid line shows the change in $R_{0}$ when the birth rates of resistant and nonresistant individuals are equivalent (scenario $1\left[\mathrm{~S}_{1}\right]$ ); the dashed line illustrates the case where the birth rate of resistant individuals is twice that of nonresistant individuals $\left(\mathrm{S}_{2}\right)$. 
congruent results from our cross-sectional and experimental studies strongly support the conclusion that nematode infection alters host Th1 function in buffalo, in a manner that may lead to variation in individual susceptibility to intracellular microparasites.

While our experimental results show a clear effect of anthelminthic treatment on baseline IFN $\gamma$ as well as body condition, we found no effect of treatment on nematode FEC. This is likely a function of the interval between the experimental treatment and recapture. The long-lasting fenbendazole bolus we used in this study suppresses nematode infection in buffalo for approximately 160 days, with $50 \%$ of treated animals showing signs of reinfection after this time period (V. O. Ezenwa and A. E. Jolles, unpublished data). Because the interval between treatment and recapture in our study was greater than 200 days, rapid nematode recolonization in treated animals may have reversed any treatment-induced differences in FEC. Nevertheless, the existence of an effect on both baseline IFN $\gamma$ and body condition suggests that nematode removal had a direct effect on host physiology. Indeed, one possible alternative explanation of our findings is that the enhanced body condition in response to nematode treatment accounted for the changes in baseline IFN $\gamma$. However, we found that differences in pre- and posttreatment baseline IFN $\gamma$ levels were driven by treatment status and not by body condition. Another possible alternative explanation for the treatment effect is that the treatment itself increased baseline IFN $\gamma$ levels. Several anthelminthic drugs have been reported as having immunomodulatory effects; however, current evidence from rodent models suggests that fenbendazole, the drug used in our study, has no effect on many standard measures of immune function (Villar et al. 2007). In sheep, where these effects have also been studied, fenbendazole treatment has been associated with reduced lymphocyte proliferation (Cabaj et al. 1994), but such suppressive effects should have resulted in a decrease, not increase, in cytokine levels in treated animals in our case. As such, it seems unlikely that either the direct effects of the treatment or the indirect effects of treatment on body condition can account for our results.

Because IFN $\gamma$ is critical for controlling $\mathrm{TB}$ infection (Flynn et al. 1993; Vordermeier et al. 2002; Welsh et al. 2005), the nematode-associated immune suppression we describe may have particularly strong effects on TB susceptibility in uninfected buffalo. Helminth infection may also enhance the progression of microparasitic diseases such as TB in infected hosts, although results to date have been equivocal (Druilhe et al. 2005; Graham et al. 2005). For example, some human studies suggest that helminth coinfection can exacerbate TB disease via persistent suppression of IFN $\gamma$ production (Elias et al. 2006; Co et al. 2007), while other studies report no correlation between
TB progression and helminth infection (Brown et al. 2006). The ability to control Mycobacterium bovis infection is associated with the maintenance of a strong IFN $\gamma$ response (Welsh et al. 2005), and this principle forms the basis of IFN $\gamma$-based TB diagnostics (Wood and Jones 2001; Schiller et al. 2009). The large difference in IFN $\gamma$ PPDB responses observed between TB-positive and TB-negative buffalo in our study demonstrates that TB-infected individuals mounted much stronger antigen-specific responses to $M$. bovis than did uninfected individuals. We used variation in IFN $\gamma$ PPDB responses among TB-positive animals as an index of the degree to which these hosts responded to disease proliferation, and we found that after accounting for severity of TB infection, TB-infected individuals with lower FECs had marginally weaker IFN $\gamma$ PPDB responses, suggesting that among TB positives, better control of nematode infection may be associated with depressed Th1 function and decreased ability to control TB. Since increased TB pathology enhances bacterial shedding (Welsh et al. 2005), the consequences of poor TB control in low-FEC animals could include faster disease progression and increased infectiousness, both of which can alter TB dynamics via effects on transmission. Body condition also had a weak effect on the host IFN $\gamma$ PPDB response, but TB severity was by far the most important factor influencing the magnitude of this response. Buffalo with more severe $\mathrm{TB}$ infections mounted a much stronger response to $\mathrm{PPDB}$ challenge, and this is consistent with work in cattle showing that animals with more severe pathology produce more IFN $\gamma$ in response to antigen stimulation (Vordermeier et al. 2002). Overall, the weak association we found between nematode FEC and IFN $\gamma$ PPDB responses in TB-positive individuals makes it difficult to draw general conclusions about nematode effects on TB progression in this system, but it is suggestive of a continuing effect of nematodes on Th1 immunity after a microparasitic infection has been acquired.

Our experimental results provide evidence that Th1 immune suppression in buffalo can be induced by nematode exposure since the effect was ameliorated by anthelminthic treatment, but this does not preclude the existence of a constitutive bias toward either a Th2 or a Th1 response. In mouse studies, nematode-resistant genotypes often show characteristically dominant Th2 responses, while nonresistant genotypes show Th1 dominance (Else and Finkelman 1998; Grencis 2001). If such genotypic variation in nematode resistance occurs in buffalo, then nematode infection may induce much stronger Thl suppression in animals that are constitutively more resistant to these parasites. The positive association we saw between FEC and baseline IFN $\gamma$ in TB negatives is suggestive of such an effect. In addition, we found direct evidence of genotypic variation in resistance to nematodes. A polymorphism at 
the BL4 microsatellite locus located upstream of the IFN $\gamma$ gene was associated with significant variation in FEC. Polymorphisms in markers at or near the IFN $\gamma$ gene have been previously linked to nematode resistance in domestic ungulates (Coltman et al. 2001; Sayers et al. 2005), and in our case, the presence of a single allele, BL4-169, was associated with a $60 \%$ increase in untransformed FEC. Interestingly, despite what appears to be a somewhat large negative effect of this allele in terms of nematode FEC, it was the second most common allele at the BL4 locus in buffalo (frequency $=18 \%$ ). The relative commonness of this allele in the face of possible negative fitness costs suggests that alleles associated with susceptibility to nematodes may be maintained in the population because they confer an advantage in terms of microparasite resistance. Our data showing that buffalo with nematode nonresistant phenotypes had significantly higher baseline IFN $\gamma$ levels provide preliminary evidence that such an advantage may exist. This type of allelic variation can be maintained by balancing selection, which is thought to be relatively common at disease resistance genes (Mitchell-Olds et al. 2007).

Our disease model explored the epidemiological outcomes of the individual-level immunological trade-offs we observed empirically. In the model, nematode exposure enhanced TB transmission as a result of Th1 immune suppression, and changes in the magnitude of the $\mathrm{TB}$ transmission rate depended on the host response to worm infection, which was determined by genotype. Irrespective of the relative proportion of nematode-resistant and nonresistant individuals in the host population, varying the nematode transmission rate- and thus the rate at which buffalo become exposed to worms-had a strong effect on the $R_{0}$ of TB. In the absence of nematodes, TB failed to invade the buffalo population; thus, under our model conditions, TB establishment in the system depended on nematodes. To our knowledge, this is the first empirical study of a natural population linking helminth coinfection to a key epidemiological parameter underpinning disease emergence. At our study site, where TB is an introduced pathogen, this points to a possible role for endemic nematodes in facilitating the successful invasion and establishment of TB in the system. In our model, a minimum increase in the TB transmission rate of 50\% among nematode-infected buffalo was sufficient to facilitate TB invasion (see fig. A2). This result highlights the fact that what can appear to be a relatively weak effect of nematodes on host Th1 immune function may translate into significant epidemiological effects in terms of microparasite dynamics. More generally, our results suggest that macroparasites may act in a manner similar to other key factors (e.g., seasonality) that can help tip the balance in favor of microparasite invasion and emergence in natural populations.
If nematode-resistant hosts experience greater Th1 suppression when exposed to nematodes, underlying genetic variation in the buffalo population might further influence the effect of nematodes on TB's $R_{0}$. We explored this possibility with our disease model and found that TB achieved a slightly higher maximum $R_{0}$ with a larger fraction of nematode-resistant genotypes in the population. Since the proportion of infected individuals in a host population increases with $R_{0}$, our results suggest that the relative frequency of nematode-resistant genotypes in the buffalo population could potentially affect TB prevalence. However, the effect of genotype frequency on $R_{0}$ was not large, suggesting that inducible immune trade-offs and not fixed genetic predisposition may be the more important mechanism linking within-host immune responses to population-level TB patterns in our system. Nevertheless, our results do raise the general issue of the potentially unforeseen consequences that can arise from breeding for nematode resistance. In livestock, artificial selection for helminth resistance is considered to be a sustainable and economical means of controlling these parasites (Sonstegard and Gasbarre 2001); however, if trade-offs in immunity are enhanced in nematode-resistant hosts, widespread breeding for resistance could exacerbate microparasite disease issues (Graham et al. 2007).

Although simple, our disease model provides a general framework for understanding the extent to which withinhost processes may influence population-level patterns of disease in the buffalo system. Model structure was determined by our current empirical understanding of the study system, and future extensions will examine how key biological complexities modify the relationship between nematode transmission and TB invasion potential. For example, we did not track individual nematode burdens in the current model, but hosts with high nematode burdens may contribute differentially to nematode transmission on the one hand and suffer increased mortality when coinfected with TB on the other. Such heterogeneities in nematode transmission coupled with differential mortality may alter system dynamics. Indeed, a recent study examining the theoretical outcomes of macroparasitemicroparasite coinfection found that while increased microparasite transmission due to coinfection can facilitate microparasite persistence, increased mortality of coinfected hosts makes microparasite persistence less likely (Fenton 2008). Our disease model considered both of these processes (increased TB transmission via nematodeinduced immune suppression and increased coinfected mortality) simultaneously, and we found a net facilitative effect of nematodes on TB. However, when we evaluated the independent effects of the two processes, we saw that the immune effect increased TB's $R_{0}$, while the ecological effect decreased $R_{0}$, consistent with the findings of Fenton 
(2008; see fig. A4 in the online edition of the American Naturalist). Future studies will explore how accounting for individual worm burdens, coupling nematode exposure to buffalo population size, and varying nematode virulence and life history (e.g., host specificity) might influence the relative magnitude of these opposing effects of coinfection. In addition, our empirical data on TB-positive hosts raise the possibility that TB progression may be accelerated in hosts with low FECs. If this is the case, these TB-positive individuals may be more infectious, die faster, or both. To address this issue as well as potential reciprocal effects of $\mathrm{TB}$ on nematodes, we will use targeted empirical studies on the direct links between nematodes, TB progression, and infectiousness to parameterize more detailed disease dynamic models.

How helminths influence host responses to microparasites and the consequences of these responses for infectious disease dynamics are emerging as important research topics in medicine, public health, epidemiology, and disease ecology (Hotez et al. 2006; Graham et al. 2007; Bradley and Jackson 2008; Walson et al. 2008). Both human and lab animal studies have elucidated key immunological mechanisms that can drive interactions between helminths and intracellular microparasites, but few studies have investigated the degree to which these mechanisms operate in wild animal populations and their ecological consequences. Here, we documented the effects of gastrointestinal nematodes on immune function in free-ranging African buffalo and described consequences for TB invasion. Given the ubiquitous nature of helminth parasitism in the wild (Nunn et al. 2003; Ezenwa et al. 2006), the processes we describe in this study are likely relevant for understanding microparasite dynamics and emergence patterns across a wide range of taxa.

\section{Acknowledgments}

Buffalo capture was conducted by KwaZulu-Natal (KZN) Wildlife and the KZN State Veterinary Service; in particular, we thank N. Armour, D. Cooper, A. McCall, W. McCall, S.-M. Ras, C. Reed, and S. van Rensburg. We also thank J. Britt, F. Gardipee, K. Kanapeckas, M. Matokazi, and M. O'Brien for assistance in the field. This work was supported by National Science Foundation (NSF) DEB 0541762/0541981, NSF EF 0723918/0723928, and a University of Montana Faculty Research Grant. R.S.E. was supported by a grant from Nederlandse Organisatie voor Wetenschappelijk Onderzoek Aard-en Levenswetenschappen (NWO-ALW).

\section{Literature Cited}

Abbas, A. K., K. M. Murphy, and A. Sher. 1996. Functional diversity of helper T lymphocytes. Nature 383:787-793.
Abu-Raddad, L. J., P. Patnaik, and J. G. Kublin. 2006. Dual infection with HIV and malaria fuels the spread of both diseases in subSaharan Africa. Science 314:1603-1606.

Ayash-Rashkovsky, M., A. L. Chenine, L. N. Steele, S. J. Lee, R. J. Song, H. Ong, R. A. Rasmussen, et al. 2007. Coinfection with Schistosoma mansoni reactivates viremia in rhesus macaques with chronic simian-human immunodeficiency virus clade $\mathrm{C}$ infection. Infection and Immunity 75:1751-1756.

Bengis, R. G. 1999. Tuberculosis: zoo and wild animal medicine. Saunders, Philadelphia.

Bentwich, Z., A. Kalinkovich, Z. Weisman, G. Borkow, N. Beyers, and A. D. Beyers. 1999. Can eradication of helminthic infections change the face of AIDS and tuberculosis? Immunology Today 20: 485-487.

Bleay, C., C. P. Wilkes, S. Paterson, and M. Viney. 2007. Densitydependent immune responses against the gastrointestinal nematode Strongyloides ratti. International Journal for Parasitology 37: 1501-1509.

Bradley, J. E., and J. A. Jackson. 2008. Measuring immune system variation to help understand host-pathogen community dynamics. Parasitology 135:807-823.

Brady, M. T., S. M. O'Neill, J. P. Dalton, and K. H. G. Mills. 1999. Fasciola hepatica suppresses a protective Th1 response against Bordetella pertussis. Infection and Immunity 67:5372-5378.

Brown, M., G. Miiro, P. Nkurunziza, C. Watera, M. A. Quigley, D. W. Dunne, J. A. G. Whitworth, and A. M. Elliott. 2006. Schistosoma mansoni, nematode infections, and progression to active tuberculosis among HIV-1-infected Ugandans. American Journal of Tropical Medicine and Hygiene 74:819-825.

Cabaj, W., M. Stankiewicz, W. E. Jonas, and L. G. Moore. 1994. Fenbendazole and its effect on the immune-system of the sheep. New Zealand Veterinary Journal 42:216-220.

Co, T. R., C. S. Hirsch, Z. Toossi, R. Dietze, and R. Ribeiro-Rodrigues. 2007. Intestinal helminth co-infection has a negative impact on both anti-Mycobacterium tuberculosis immunity and clinical response to tuberculosis therapy. Clinical and Experimental Immunology 147:45-52.

Coltman, D. W., K. Wilson, J. G. Pilkington, M. J. Stear, and J. M. Pemberton. 2001. A microsatellite polymorphism in the gamma interferon gene is associated with resistance to gastrointestinal nematodes in a naturally-parasitized population of Soay sheep. Parasitology 122:571-582.

Druilhe, P., A. Tall, and C. Sokhna. 2005. Worms can worsen malaria: towards a new means to roll back malaria? Trends in Parasitology 21:359-362.

Elias, D., G. Mengistu, H. Akuffo, and S. Britton. 2006. Are intestinal helminths risk factors for developing active tuberculosis? Tropical Medicine and International Health 11:551-558.

Else, K. J., and F. D. Finkelman. 1998. Intestinal nematode parasites, cytokines and effector mechanisms. International Journal for Parasitology 28:1145-1158.

Ezenwa, V. O. 2003. Habitat overlap and gastrointestinal parasitism in sympatric African bovids. Parasitology 126:379-388.

Ezenwa, V. O., S. A. Price, S. Altizer, N. D. Vitone, and K. C. Cook. 2006. Host traits and parasite species richness in even and oddtoed hoofed mammals, Artiodactyla and Perissodactyla. Oikos 115: 526-536.

Ezenwa, V. O., A. E. Jolles, and M. P. O'Brien. 2009. A reliable body condition scoring technique for estimating condition in African buffalo. African Journal of Ecology 47:476-481. 
Fenton, A. 2008. Worms and germs: the population dynamic consequences of microparasite-macroparasite co-infection. Parasitology 135:1545-1560.

Finkelman, F. D., T. Shea-Donohue, J. Goldhill, C. A. Sullivan, S. C. Morris, K. B. Madden, W. C. Gause, and J. F. Urban Jr. 1997. Cytokine regulation of host defense against parasitic gastrointestinal nematodes: lessons from studies with rodent models. Annual Review of Immunology 15:505-533.

Flynn, J. L., J. Chan, K. J. Triebold, D. K. Dalton, T. A. Stewart, and B. R. Bloom. 1993. An essential role for interferon $\gamma$ in resistance to Mycobacterium tuberculosis infection. Journal of Experimental Medicine 178:2249-2254.

Gasbarre, L. C., E. A. Leighton, and T. Sonstegard. 2001. Role of the bovine immune system and genome in resistance to gastrointestinal nematodes. Veterinary Parasitology 98:51-64.

Graham, A. L., T. J. Lamb, A. F. Read, and J. E. Allen. 2005. Malariafilaria coinfection in mice makes malarial disease more severe unless filarial infection achieves patency. Journal of Infectious Diseases 191:410-421.

Graham, A. L., I. M. Cattadori, J. O. Lloyd-Smith, M. J. Ferrari, and O. N. Bjornstad. 2007. Transmission consequences of coinfection: cytokines writ large? Trends in Parasitology 23:284-291.

Grencis, R. K. 2001. Cytokine regulation of resistance and susceptibility to intestinal nematode infection: from host to parasite. Veterinary Parasitology 100:45-50.

Grobler, D. G., A. L. Michel, L. M. DeKlerk, and R. G. Bengis. 2002. The gamma-interferon test: its usefulness in a bovine tuberculosis survey in African buffaloes (Syncerus caffer) in the Kruger National Park. Onderstepoort Journal of Veterinary Research 69:221-227.

Hotez, P. J., D. H. Molyneux, E. Stillwaggon, Z. Bentwich, and J. Kumaresan. 2006. Neglected tropical diseases and HIV/AIDS. Lancet 368:1865-1866.

Jolles, A. E., D. V. Cooper, and S. A. Levin. 2005. Hidden effects of chronic tuberculosis in African buffalo. Ecology 86:2358-2364.

Jolles, A. E., V. O. Ezenwa, R. S. Etienne, W. C. Turner, and H. Olff. 2008. Interactions between macroparasites and microparasites drive infection patterns in free-ranging African buffalo. Ecology 89:2239-2250.

La Flamme, A. C., P. Scott, and E. J. Pearce. 2002. Schistosomiasis delays lesion resolution during Leishmania major infection by impairing parasite killing by macrophages. Parasite Immunology 24: 339-345.

Lloyd-Smith, J. O., M. Poss, and B. T. Grenfell. 2008. HIV-1/parasite co-infection and the emergence of new parasite strains. Parasitology 135:795-806.

Maizels, R. M., A. Balic, N. Gomez-Escobar, M. Nair, M. D. Taylor, and J. E. Allen. 2004. Helminth parasites: masters of regulation. Immunological Reviews 201:89-116.

Michel, A. L., R. G. Bengis, D. F. Keet, M. Hofmeyr, L. M. de Klerk, P. C. Cross, A. E. Jolles, et al. 2006. Wildlife tuberculosis in South African conservation areas: implications and challenges. Veterinary Microbiology 112:91-100.

Mitchell-Olds, T., J. H. Willis, and D. B. Goldstein. 2007. Which evolutionary processes influence natural genetic variation for phenotypic traits? Nature Reviews Genetics 8:845-856.

Mosmann, T. R., and S. Sad. 1996. The expanding universe of T-cel subsets: Th1, Th2 and more. Immunology Today 17:138-146.

Murphy, K. M., P. Travers, and M. Walport. 2007. Janeway's immunobiology. 7th ed. Garland Science, London.
Nunn, C. L., S. Altizer, K. E. Jones, and W. Sechrest. 2003. Comparative tests of parasite species richness in primates. American Naturalist 162:597-614.

O'Neill, S. M., K. H. Mills, and J. P. Dalton. 2000. Fasciola hepatica infection downregulates Th1 responses in mice. Parasite Immunology 22:147-155.

Poulin, R. 2007. Evolutionary ecology of parasites. 2nd ed. Princeton University Press, Princeton, NJ.

Raymond, M., and F. Rousset. 1995. Genepop version 1.2: population-genetics software for exact tests and ecumenicism. Journal of Heredity 86:248-249.

Sayers, G., B. Good, J. P. Hanrahan, M. Ryan, and T. Sweeney. 2005. Intron 1 of the interferon gamma gene: its role in nematode resistance in Suffolk and Texel sheep breeds. Research in Veterinary Science 79:191-196.

Schiller, I., W. R. Waters, H. M. Vordermeier, B. Nonnecke, M. Welsh, N. Keck, A. Whelan, et al. 2009. Optimization of a whole-blood gamma interferon assay for detection of Mycobacterium bovis infected cattle. Clinical and Vaccine Immunology 16:1196-1202.

Sinclair, A. R. E. 1977. The African buffalo: a study of resource limitation of populations. University of Chicago Press, Chicago.

Sonstegard, T. S., and L. C. Gasbarre. 2001. Genomic tools to improve parasite resistance. Veterinary Parasitology 101:387-403.

Stear, M. J., L. Fitton, G. T. Innocent, L. Murphy, K. Rennie, and L. Matthews. 2007. The dynamic influence of genetic variation on the susceptibility of sheep to gastrointestinal nematode infection. Journal of the Royal Society Interface 4:767-776.

Su, Z., M. Segura, K. Morgan, J. C. Loredo-Osti, and M. M. Stevenson. 2005. Impairment of protective immunity to blood-stage malaria by concurrent nematode infection. Infection and Immunity 73:3531-3539.

Telfer, S., R. Birtles, M. Bennett, X. Lambin, S. Paterson, and M. Begon. 2008. Parasite interactions in natural populations: insights from longitudinal data. Parasitology 135:767-781.

Villar, D., C. Cray, J. Zaias, and N. H. Altman. 2007. Biologic effects of fenbendazole in rats and mice: a review. Journal of the American Association for Laboratory Animal Science 46:8-15.

Vordermeier, H. M., M. A. Chambers, P. J. Cockle, A. O. Whelan, J. Simmons, and R. G. Hewinson. 2002. Correlation of ESAT-6specific gamma interferon production with pathology in cattle following Mycobacterium bovis BCG vaccination against experimental bovine tuberculosis. Infection and Immunity 70:30263032 .

Walson, J. L., P. A. Otieno, M. Mbuchi, B. A. Richardson, B. LohmanPayne, S. W. Macharia, J. Overbaugh, et al. 2008. Albendazole treatment of HIV-1 and helminth co-infection: a randomized, double-blind, placebo-controlled trial. AIDS 22:1601-1609.

Welsh, M. D., R. T. Cunningham, D. M. Corbett, R. M. Girvin, J. McNair, R. A. Skuce, D. G. Bryson, and J. M. Pollock. 2005. Influence of pathological progression on the balance between cellular and humoral immune responses in bovine tuberculosis. Immunology 114:101-111.

Wood, P. R., and S. L. Jones. 2001. BOVIGAM: an in vitro cellular diagnostic test for bovine tuberculosis. Tuberculosis 81:147-155.

Associate Editor: Gregory E. Demas Editor: Mark A. McPeek 
Copyright of American Naturalist is the property of University of Chicago Press and its content may not be copied or emailed to multiple sites or posted to a listserv without the copyright holder's express written permission. However, users may print, download, or email articles for individual use. 